\title{
Perfil epidemiológico dos usuários do Centro de Testagem e Aconselhamento de Alfenas, Minas Gerais
}

\author{
Epidemiological profile of the clientele in HIV Testing and Counseling Center in Alfenas, Minas Gerais
}

Perfil epidemiológico de los usuarios del Centro de Pruebas y Consejeria de Alfenas, Minas Gerais

Maraisa Pimenta Vilela ${ }^{\mathrm{I}}$, Tábatta Renata Pereira de Brito ${ }^{\mathrm{II}}$, Sueli Leiko Takamatsu GoyatáiII ${ }^{\text {, }}$ Cássia Irene Spinelli Arantes ${ }^{\mathrm{IV}}$ ${ }^{\text {I }}$ Aluna do curso de graduação em Enfermagem, Universidade Federal de Alfenas (UNIFAL-MG). Alfenas, MG, Brasil: E-mail: maraisa_vilela@yahoo.com.br.

II Aluna do curso de graduação em Enfermagem, UNIFAL-MG. Alfenas, MG, Brasil: E-mail: tabatta renata@hotmail.com.

III Enfermeira, Doutora em Enfermagem. Professor Adjunto, Departamento de Enfermagem, UNIFAL-MG. Alfenas, MG, Brasil: E-mail: sueligoyata@yahoo.com.br.

IV Enfermeira, Doutora em Enfermagem. Professor Associado, Departamento de Enfermagem, UNIFAL-MG. Alfenas, MG, Brasil: E-mail: cassiaarantes@terra.com.br.

\section{RESUMO}

O Centro de Testagem e Aconselhamento (CTA) foi instituído para atuar no diagnóstico e prevenção de infecções sexualmente transmissíveis (IST) e constitui-se em importante fonte de dados epidemiológicos. Com o objetivo de identificar o perfil epidemiológico dos usuários do CTA no município de Alfenas-MG foi realizado um estudo transversal, descritivo e exploratório, no período de agosto de 2007 a junho de 2008. Foram utilizados dados secundários dos usuários cadastrados no serviço, que foram armazenados e analisados por meio do Programa Epi-info versão 6.04. No total, foram realizados 162 atendimentos, com predomínio de pessoas do sexo feminino $(53,1 \%)$ e média de idade de 35,9 anos. As taxas de positividade para o HIV, hepatites B e C e sífilis foram 1,9\%, 14,4\%,5,9\% e 1,9\%, respectivamente. O principal motivo de procura pelo CTA foi a exposição à situação de risco $(43,8 \%)$. O estudo evidenciou que, por meio das ações do CTA, é possível monitorar o status sorológico, o perfil sociodemográfico e os comportamentos relacionados ao HIV/Aids e às outras IST na clientela atendida, o que contribui para o alcance dos objetivos relacionados a prevenção e controle dessas doenças. Descritores: Síndrome da Imunodeficiência Adquirida; Doenças sexualmente transmissíveis; Perfil de saúde; Aconselhamento.

\section{ABSTRACT}

The Center Testing and Counseling (CTA) was established to act in the diagnosis and prevention of sexually transmitted infections (STI) and it constituted an important source of epidemiological data. With the objective of identifying the epidemiological profile of the clientele in CTA in Alfenas City, Minas Gerais, a cross-sectional study, descriptive and exploratory was carried out, in the period of August 2007 to June 2008. Secondary data of the clientele registered in the service, that were stored and analyzed through the program Epi-info, version 6.04. In the total, 162 services were accomplished, with feminine gender people prevalence $(53.1 \%)$ and average of 35.9 years-old. The positiveness taxes for HIV, hepatitis B and C and syphilis were respectively, $1.9 \%, 14.4 \%, 5.9 \%$ and $1.9 \%$. The main reason for seeking the CTA was the exhibition to the risk situation (43.8\%). The study evidenced that through the actions of CTA is possible to monitor the status serology and the social demographic profile and behavior related to HIV/Aids and other STI in the clientele, what contributes to the achievement of objectives related to prevention and control of these diseases.

Descriptors: Acquired Immunodeficiency Syndrome; Sexually Transmitted Diseases; Health Profile; Counseling.

\section{RESUMEN}

El Centro de Pruebas y Consejeria (CTA) fue instituído para actuar en el diagnóstico y prevención de infecciones sexualmente transmisibles (ETS) y constituirse como importante fuente de datos epidemiológicos. Con el objetivo de identificar el perfil epidemiológico de los usuarios del CTA del município de Alfenas, Minas Gerais, se realizó un estudio transversal, descriptivo y exploratório, en el periodo de agosto de 2007 a junio de 2008. Fueron utilizados datos secundarios de los inscritos en el servicio, mismos que fueron almacenados y analizados por medio del programa Epi-info,versión 6.04.En total realizaronse 162 atendimientos, predominando mujeres $(53,1 \%)$ con media de edad de 35,9 años. Las tasas de positividad para HIV, hepatitis B Y C y sífilis fueron respectivamente: 1,9\%, 14,4\%,5,9\% y 1,9\%. El principal motivo para la procura del CTA fue la exposición a situaciones de riesgo $(43,8 \%)$. El estudio evidenció que a través de las acciones del CTA es posible acompañar el estatus serológico y el perfil sociodemográfico y de comportamiento relacionados al HIV/SIDA y a otras ETS en las personas atendidas, lo que contribuye para alcanzar los objetivos relacionados a la prevención y control de estas enfermedades.

Descriptores: Síndrome de Inmunodeficiencia Adquirida; Enfermedades de Transmisión Sexual; Perfil de la Salud; Consejeria. 


\section{INTRODUÇÃO}

O Brasil é o país que apresenta o maior número absoluto de casos da síndrome da imunodeficiência adquirida (Aids) na América Latina, sendo que de 1,8 milhões de pessoas portadoras do vírus da imunodeficiência humana (HIV), um terço delas vivem no País(1).

A Aids, como é conhecida mundialmente, trouxe para a população brasileira sentimentos de medo, pânico e preconceito a partir da identificação dos primeiros casos, no início da década de $80^{(1)}$. Progressivamente essa doença passou a ser reconhecida como fenômeno social, após a desmistificação da idéia de que acometia somente grupos restritos de risco, como, por exemplo, os homossexuais e os hemofílicos $^{(2)}$. Assim, a evolução da epidemia de Aids no Brasil tem apresentado novo curso em que se destacam a pauperização, a feminilização, a juvenilização e a interiorização como resultado das profundas desigualdades da sociedade brasileira ${ }^{(3-7)}$. Além disso, tem-se observado uma tendência de desaceleração a partir de 1997, o que não ocorreu de forma homogênea nas macrorregiões do país. Essas diferenças podem ser atribuídas, entre outras, às medidas de prevenção implementadas, ao conhecimento da população sobre a Aids e ao acesso à terapia antiretroviral $(T A R V)^{(1)}$. A esse respeito, a terapia antiretroviral, a partir de 1996, aumentou o tempo da sobrevida e do quadro clínico-laboratorial e reduziu as internações das pessoas que vivem com HIV/Aids ${ }^{(3)}$.

Em relação à vigilância da infecção pelo HIV e da Aids no Brasil, o Ministério da Saúde prevê a notificação universal dos casos, a vigilância sorológica em populaçõessentinela, as estimativas sorológicas e/ou comportamentais de base populacional em populações específicas e em Centros de Testagem e Aconselhamento (CTA) ${ }^{(1)}$.

Os CTA, anteriormente denominados Centros de Orientação e Apoio Sorológico (COAS) ${ }^{(4)}$, são atualmente definidos como serviços de saúde que realizam ações de diagnóstico e de prevenção de infecções sexualmente transmissíveis (IST), além de constituir importante fonte de dados, permitindo investigar e descrever o perfil epidemiológico dos usuários, orientando medidas específicas de prevenção ${ }^{(8-9)}$. Nesses serviços, é possível realizar testes para HIV, sífilis e hepatites B e C gratuitamente. Todos os exames são feitos de acordo com a norma definida pelo Ministério da Saúde e com produtos registrados na Agência Nacional de Vigilância Sanitária (ANVISA/MS) e por ela controlados. $\mathrm{O}$ atendimento é inteiramente sigiloso e oferece a quem realiza o teste, a possibilidade de ser acompanhado por uma equipe de profissionais de saúde, que orientará sobre o resultado final do exame, independentemente de ele ser positivo ou negativo. As ações de aconselhamento realizadas nos CTA visam informar os usuários sobre HIV/Aids e orientá-los em relação às medidas de prevenção e ao enfrentamento à soropositividade e à doença ${ }^{(10)}$.

Em Alfenas-MG, o CTA foi criado em 2003 e desde então passou por várias coordenações, o que provocou certa descontinuidade dos programas implantados e dificuldades na assistência prestada aos usuários. O preenchimento sistemático dos formulários de atendimento aos usuários do CTA, realizado de forma manual, teve início em agosto de 2007 e até o momento não foi informatizado.

Considerando que o CTA se configura como um serviço estratégico para a produção de conhecimento voltado às tendências da epidemia de Aids nos diferentes níveis de atenção à saúde, e que em Alfenas-MG havia a necessidade de formulação de estratégias para intensificar e fortalecer as ações desse serviço, realizou-se um estudo que teve como objetivo identificar o perfil epidemiológico dos usuários que buscaram por atendimento no CTA do município, segundo as características sociodemográficas, comportamentos de risco e positividade para HIV, hepatites virais $B$ e $C$ e sífilis.

\section{MÉTODO}

Foi realizado um estudo retrospectivo transversal a partir das informações coletadas de fonte secundária ${ }^{(11)}$, utilizando o Formulário de Cadastro dos Usuários que buscaram por atendimento no CTA do município de AlfenasMG, durante o período de agosto de 2007 a junho de 2008.

As variáveis selecionadas para este estudo foram o sexo, a faixa etária, o estado civil, a escolaridade, a raça/cor, a situação ocupacional, a cidade de origem, a localização geográfica e territorial dos usuários, o motivo por procurar o serviço, a forma de divulgação do CTA, a procura pelo banco de sangue para testagem, o consumo de drogas, o tipo de parceiro sexual, o tipo de exposição ao HIV, o uso de preservativo e a soropositividade para HIV, hepatites $B$ e $C$ e sífilis.

A tabulação e a análise dos dados foram feitas no software Epi-info versão 6.04, em que se calculou a média e o desvio-padrão. Os resultados foram mostrados em gráficos e tabelas.

Esta pesquisa foi aprovada pelo Comitê de Ética em Pesquisa da Universidade Federal de Alfenas-MG, após a autorização da Coordenação do CTA de Alfenas-MG e da Secretaria Municipal de Saúde, sendo aprovada em 30 de junho de 2008, protocolo $n^{\circ}$ 23087.001167/2008-46, segundo as recomendações da Resolução 196/96 ${ }^{(12)}$.

\section{RESULTADOS E DISCUSSÃO}

Foram realizados 162 atendimentos com média de 16,2 atendimentos/mês, nas consultas de aconselhamento individual no CTA de Alfenas-MG. De todas as pessoas que passaram por atendimento, 94,4\% fizeram a coleta de material para exames, sendo que os resultados não estavam disponíveis em $10,5 \%$ dos formulários. Uma provável explicação para a ausência dos resultados de exames nos devidos campos pode estar na falta de retroalimentação do registro no formulário logo que a equipe recebe o laudo do laboratório.

Houve predominância de usuários do sexo feminino $(53,1 \%)$. Esse resultado era esperado, uma vez que as mulheres tendem a procurar por atendimento de saúde com maior frequência, pela oferta de serviços e maior disponibilidade de tempo atribuído a essa clientela(13).

O Gráfico 1 apresenta a distribuição percentual dos usuários que buscaram atendimento no CTA de Alfenas-MG, por faixa etária. 
Gráfico 1: Distribuição percentual dos usuários que buscaram atendimento no CTA, por faixa etária. Alfenas,MG, 2008.

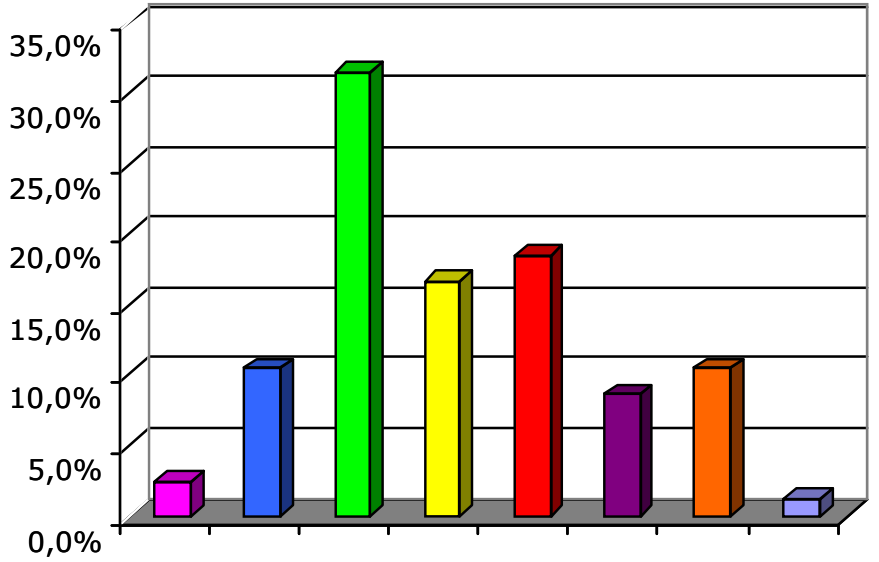

1 a 14

15 a 19

20 a 29

$\square 30$ a 39

40 a 49

50 a 59

mais 60

sem registro
Houve predominância da faixa etária de 20 a 29 anos $(33,9 \%)$ e a média de idade foi de 35,9 anos, desvio padrão $\pm 15,6$, variando de 11,9 a 79,6 anos de idade. Resultado semelhante foi encontrado em estudo realizado no CTA do Hospital Escola São Francisco de Assis (CTA-HESFA), localizado no Rio de Janeiro, no qual a faixa etária predominante de atendimento foi a de 20 a $29 \operatorname{anos}^{(14)}$. No CTA da cidade de Fortaleza-CE, também houve predominância da população adulta jovem, na faixa etária de 19 a 30 anos $^{(12)}$.

Em relação ao estado civil, 54,3\% dos usuários eram solteiros e $31,5 \%$ casados ou em situação de união estável. Esses achados diferem dos resultados encontrados no estudo de Griep e colaboradores ${ }^{(14)}$, no qual $44,4 \%$ eram casados ou em situação de união estável e $41,5 \%$ eram solteiros.

Dos usuários que buscaram por atendimento no CTA de Alfenas, $32,1 \%$ apresentaram 12 anos ou mais de estudo. Em pesquisa realizada por Griep e colaboradores ${ }^{(14)}, 45,7 \%$ haviam estudado de quatro a sete anos. A escolaridade parece ter o seu destaque reduzido nas práticas de risco ao HIV uma vez que, independente de escolaridade, atualmente a população brasileira tem tido acesso considerável à informação básica sobre as formas de transmissão do vírus. Entretanto, o acesso aos meios de prevenção e de tratamento das IST está diretamente relacionado ao nível educacional institucional ${ }^{(8)}$.

Analisando a raça/cor, 50,0\% relataram serem brancos; 21,0\%, pardos; 6,8\%, negros; 0,6\%, amarelos; $1,2 \%$, indígenas e $20,4 \%$ não informaram.

As ocupações mais citadas foram estudantes $(17,9 \%)$, domésticas $(17,3 \%)$, profissionais do sexo $(5,6 \%)$ e aposentados $(4,9 \%)$.

Considerando que a Gerência Regional de Saúde (GRS) de Alfenas é composta por 26 municípios e que o município de Alfenas é o único que possui CTA, é relevante o fato de que $96,5 \%$ de todos os atendimentos foram da população de Alfenas, sendo registrados apenas atendimentos às cidades de Divisa Nova e Monte Belo, localizadas na microrregião de saúde, o que correspondeu a $2,9 \%$ e $0,6 \%$ dos casos, respectivamente.

Quanto a localização geográfica e territorial, a maioria dos usuários $(31,5 \%)$ era residente da região central da cidade. Uma hipótese levantada para explicar esse fato se deve à facilidade de acesso e à proximidade entre o local de moradia e o local de atendimento ${ }^{(14)}$.
A distribuição percentual dos usuários que buscaram por atendimento no CTA de Alfenas, segundo as áreas de abrangência das unidades de saúde da família em Alfenas evidencia que a grande proporção dos usuários do serviço (42\%) é advinda de locais fora de área de abrangência dos Programas de Saúde da Família (PSF). Entre os PSF, os do Santos Reis e Jardim São Carlos, que se localizam em regiões periféricas do município, são os que apareceram com maior frequência como local de residência dos usuários, 9,9\% e 7,4\%, respectivamente.

Semelhante ao que se encontrou no CTA do Hospital Escola São Francisco de Assis ${ }^{(14)}$, a exposição à situação de risco foi a causa mais frequente pela procura de atendimento no CTA $(43,8 \%)$, seguida da prevenção $(24,1 \%)$ e testagem para hepatites $(20,0 \%)$. Esse resultado pode estar diretamente relacionado ao estado civil, tendo em vista que $54,3 \%$ dos usuários que buscaram por atendimento no CTA de Alfenas eram solteiros. Essas pessoas tendem a ter mais parceiros sexuais do que aquelas que estão em uma convivência dita estável, o que reforça o entendimento de que não somente grupos restritos são susceptíveis à infecção pelo HIV, mas que o risco está em determinados comportamentos, os quais aumentam as chances de sua transmissão(15-16).

A forma de divulgação da testagem oferecida pelo CTA entre os usuários é diversificada, sendo que jornais, rádio e televisão $(22,8 \%)$ e material de divulgação específico $(22,8 \%)$ apareceram em destaque. Germano e colaboradores $^{(8)}$ afirmam que os CTA têm um importante papel na estabilização da epidemia de Aids e que campanhas de esclarecimento sobre sua existência deveriam ser intensificadas, devido à importância da testagem sorológica, do conhecimento do diagnóstico e das modalidades de transmissão e prevenção ao HIV.

Em relação à procura pelo banco de sangue para testagem, encontrou-se que $82,7 \%$ dos usuários não faziam uso desse serviço de saúde.

A Tabela 1 apresenta a distribuição percentual dos usuários que buscaram por atendimento no CTA de AlfenasMG, em relação ao tipo e à frequência do uso de drogas utilizadas pelos usuários. 
Tabela 1: Distribuição percentual das drogas usadas pelos usuários do CTA em relação ao tipo e à frequência de uso. Alfenas, MG, 2008.

\begin{tabular}{|c|c|c|c|c|c|c|}
\hline Frequência do uso & $\begin{array}{c}\text { Álcool } \\
(\%)\end{array}$ & $\begin{array}{c}\text { Maconha } \\
(\%)\end{array}$ & $\begin{array}{c}\text { Cocaína Aspirada } \\
(\%)\end{array}$ & $\begin{array}{c}\text { Cocaína Injetável } \\
(\%)\end{array}$ & $\begin{array}{l}\text { Heroína } \\
(\%)\end{array}$ & $\begin{array}{c}\text { Anfetaminas } \\
(\%)\end{array}$ \\
\hline Nunca usou & 63,6 & 86,4 & 92,0 & 92,0 & 92,6 & 92,5 \\
\hline $\begin{array}{c}\text { Já usou, mas não usa } \\
\text { mais }\end{array}$ & 4,9 & 4,9 & 1,2 & 1,2 & 1,2 & 1,3 \\
\hline Usa de vez em quando & 20,4 & 1,2 & 0,0 & 0,0 & 0,0 & 0,0 \\
\hline Usa frequentemente & 4,9 & 1,2 & 0,6 & 0,6 & 0,0 & 0,0 \\
\hline Sem registro & 6,2 & 6,2 & 6,2 & 6,2 & 6,2 & 6,3 \\
\hline
\end{tabular}

O uso esporádico de álcool é informado por $20,4 \%$ dos usuários. Entre as drogas ilícitas, a maconha é a mais citada pelos indivíduos $(7,0 \%)$, sendo que $1,2 \%$ não usam mais; $1,2 \%$ usam esporadicamente e esse mesmo percentual usa frequentemente. Apenas $0,6 \%$ afirmou que fazia uso frequente de drogas injetáveis e $0,6 \%$ dos indivíduos referiu o compartilhamento de seringas nos últimos 12 meses.

Em relação ao tipo de parceiro sexual no último ano, homens que se relacionaram com homens foram 6,2\%; mulheres que se relacionaram com mulheres, $2,5 \%$ e relações com travestis e transexuais, 0,6\%.

No Brasil, 63,8\% dos casos de Aids diagnosticados entre 1980 e 2003 foram transmitidos por via sexual. Desde a década de 90, o maior número de casos ocorre entre os heterossexuais. Em 2003, a via sexual com mulheres foi responsável por 39,6\% das infecções em homens com 13 anos ou mais, contra $16,2 \%$ dos casos masculinos, em 1992. Enquanto a epidemia cresce entre os heterossexuais, a transmissão de Aids entre os homossexuais tem apresentado significativa redução. Em 1992, a relação sexual com pessoa do mesmo sexo foi responsável por $25,2 \%$ dos casos diagnosticados e em 2003, esse índice caiu para $15,9 \%{ }^{(17)}$.

O tipo de exposição ao HIV predominante nos registros é a relação sexual, com 46,9\%. Nessa situação, o uso de preservativos de maneira adequada seria a principal forma de se reduzir o risco de contaminação pelo $\mathrm{HIV}^{(18)}$.

A avaliação do uso de preservativo pode ser realizada em duas situações diferentes, nas relações sexuais com parceiros fixos e com parceiros eventuais. Quando se analisa a situação com parceiros fixos, observa-se que $38,5 \%$ dos usuários referiram nunca utilizar o preservativo nas relações sexuais. Assim como no estudo descrito por Szwarcwald e colaboradores ${ }^{(18)}$, os resultados evidenciaram que uma proporção maior de usuários referiu sempre fazer uso do preservativo quando se relaciona com parceiros eventuais $(12,3 \%)$ do que com parceiros fixos $(8,1 \%)$.

Os principais motivos dos usuários do CTA de AlfenasMG do não uso do preservativo durante as relações sexuais, quando se tratava de relacionamento com parceiro fixo, foram a confiança no parceiro $(23,1 \%)$ e o fato de não gostar de usar o preservativo $(18,8 \%)$. Já os motivos apontados, quando o relacionamento se referia a parceiros eventuais foram o fato de não gostar de usar (8\%) e a indisponibilidade no momento (2,5\%). Esses comportamentos agravam a situação epidemiológica, uma vez que os usuários tendem a usar o preservativo ao iniciar os relacionamentos eventuais e progressivamente abandonam essa prática preventiva na medida em que a relação passa a ser considerada, pelos parceiros, como estável ${ }^{(15)}$.

Em relação aos atendimentos realizados que culminaram com a coleta de material para exames, 1,9\% foram positivos para HIV. A frequência de indivíduos soropositivos para HIV encontrada no CTA de Alfenas-MG está em conformidade com os dados nacionais, uma vez que as taxas de positividade da população atendida nas diferentes regiões brasileiras variam de $1 \%$ a $5 \%$, nas regiões Sul e Sudeste ${ }^{(19)}$.

Do total dos usuários que buscaram por atendimento no CTA, $14,4 \%$ e $5,9 \%$ apresentaram positividade para hepatites B e C, respectivamente, e 1,9\% para sífilis. A necessidade de testagem para as hepatites e HIV se deve ao fato de ambos apresentarem semelhanças entre as formas de transmissão e de apresentarem repercussões mais graves ${ }^{(15)}$. A sífilis já ocupou um lugar de destaque entre as $\mathrm{IST}^{(8)}$. Com 0 advento da antibioticoterapia (penicilina), houve uma mudança na situação epidemiológica dessa enfermidade, possibilitando a cura, a interrupção da evolução e uma importante redução da mortalidade ${ }^{(8,20)}$.

\section{CONCLUSÃO}

O estudo possibilitou a identificação do perfil epidemiológico dos 162 usuários que buscaram por atendimento no CTA do município de Alfenas-MG, durante o período de agosto de 2007 a junho de 2008. Na procura pelo serviço, prevaleceram adultos jovens e do sexo feminino. Na análise dos resultados de exames sorológicos, encontraram-se $1,9 \%$ positivos para HIV; $14,4 \%$ e $5,9 \%$ para hepatites $\mathrm{B}$ e $\mathrm{C}$, respectivamente, e 1,9\% para sífilis. $\mathrm{O}$ principal motivo de procura pelo CTA foi a exposição à situação de risco (43,8\%); no entanto, 38,5\% dos usuários referiram nunca utilizar o preservativo nas relações sexuais. Chama a atenção o número de usuários que relatou não fazer uso de método preventivo contra a infecção pelo HIV/IST, o que indica a necessidade de mudanças nos padrões comportamentais e culturais, relacionados à prevenção desses agravos.

O CTA de Alfenas se constitui em importante recurso para saúde, uma vez que oferece o teste anti-HIV para a população dos municípios da microrregião de saúde, sobretudo para os residentes em Alfenas-MG. Por intermédio desse serviço, pode-se monitorar o status sorológico, o perfil sociodemográfico e o comportamento de risco relacionado à infecção por HIV da clientela atendida. Nesse aspecto, a implementação de um sistema municipal de informações, abrangendo os dados obtidos nas consultas de aconselhamento e no acompanhamento aos doentes de AIDS, poderia agilizar o seu monitoramento e promover a vigilância à saúde da população mais vulnerável na microrregião de Alfenas-MG.

\section{REFERÊNCIAS}

1. Dourado I, Veras MASM, Barreira D, Brito AM. Tendências da epidemia de Aids no Brasil após a terapia anti-retroviral. Rev Saude Publica. 2006;40 Suppl:18-22. 
2. Oliveira DC, Formozo GA, Gomes AMT, Acioli S, Marques SC, Costa TL et al. A produção de conhecimento sobre HIV/AIDS no campo da teoria de representações sociais em 25 anos da epidemia. Rev. Eletr. Enf. [Internet] 2007 [cited 2010 jun 30];9(3):821-34. Available from: http://www.fen.ufg.br/revista/v9/n3/v9n3a21.htm

3. Helena ETS, Mafra ML, Simes M. Fatores associados à sobrevida de pessoas vivendo com Aids no Município de Blumenau, Estado de Santa Catarina, Brasil, 1997-2004. Epidemiol. Serv. Saude. 2009;18(1):45-53.

4. Souza V, Czarisnia D, Natividade C. Aconselhamento na prevenção do HIV:olhar dos usuários de um centro de testagem. Cad Saude Publica. 2008;24(7):1536-44.

5. Schneider IJC, Ribeiro C, Breda D, Skalinski LM, D'Orsi E. Perfil epidemiológico dos usuários dos Centros de Testagem e Aconslehamento do Estado de Santa Catarina, Brasil, no ano de 2005. Cad Saude Publica. 2008;24(7):1675-8.

6. Fonseca MGP, Szwarcwald CL, Bastos FI. Análise sociodemográfica da epidemia de Aids no Brasil 1989-1997. Rev Saude Publica. 2002;36(6):678-85.

7. Parker R, Camargo Jr. KR. Pobreza e HIV/AIDS: aspectos antropológicos e sociológicos. Cad Saude Publica. 2000;16 Suppl 1:89-102.

8. Germano FN, Silva TMG, Mendoza-Sassi R, Martinez AMB. Alta prevalência de usuários que não retornam aos Centros de Testagem e Aconselhamento (CTA) para o conhecimento de seus status sorológico - Rio Grande, RS, Brasil. Cien Saude Colet. 2008;13(3):1033-40.

9. Bassicheto KC, Mesquita F, Zacaro C, Santos EA, Oliveira SM, Veras MASM et al. Perfil epidemiológico dos usuários de um Centro de Testagem e Aconselhamento para DST/HIV da Rede Municipal de São Paulo com sorologia positiva para o HIV. Rev. bras. epidemiol. 2004;7(3):302-10.

10. Ministério da Saúde. Coordenação Nacional de DST e AIDS. Diretrizes dos Centros de Testagem e Aconselhamento (CTA)- Manual. Brasília: Ministério da Saúde; 1999.

11. Pereira MG. Epidemiologia: teoria e prática. Rio de janeiro: Guanabara Koogan; 2008.

12. Ministério da Saúde; Conselho Nacional de Saúde. Resolução n 196/96 - Normas regulamentadoras de pesquisa envolvendo seres humanos. Brasília: Ministério da Saúde; 1996.

13. Loyola Filho AI, Uchoa E, Guerra HL, Firmo JOA, LimaCosta MF. Prevalência e fatores associados à automedicação: resultados do projeto Bambuí. Rev Saude Publica. 2002;36(1):55-62.

14. Griep RH, Paula LR, Almeida A, Quadra MCO. Perfil de usuários de um Centro de Testagem e Aconselhamento a partir de dados do SI-CTA. In: Ministério da Saúde; Secretaria de Vigilância em Saúde. Centro de Testagem e Aconselhamento (CTA): Integrando prevenção e assistência. Brasília: Ministério da Saúde; 2006. p. 56-67.

15. Araújo MAL, Sales AMR, Diogenes MAR. Hepaties B e C em usuários do Centro de Testagem e Aconselhamento (CTA) de Fortaleza-Ceará. DST - J bras Doenças Sex Transm. 2006;18(3):161-7.

16. Bastos FI. Aids na terceira década. Rio de Janeiro: Editora Fiocruz; 2006.

17. Ministério da Saúde. Departamento de DST, Aids e hepatites virais. Aids: transmissão entre homossexuais apresenta redução significativa. Brasília: Ministério da Saúde; 2004.

18. Szwarcwald CL, Bastos FI, Esteves MAP, Andrade CLT. A disseminação da epidemia de AIDS no Brasil, no período de
1987-1996: uma análise espacial. Cad Saude Publica. 2000;16(1):7-19.

19. Grangeiro A, Martinson B, Silva SGM, Barreira D, Ferraz $D$, Rocha $F$ et al. Diagnóstico situacional dos Centros de Testagem e Aconselhamento. São Paulo: Instituto de Saúde; 2007.

20. Lima BGC. Mortalidade por sífilis nas regiões brasileiras, 1980-1995. J. Bras. Patol. Med. Lab.2002;38(4):267-71.

Artigo recebido em 30.05.2009.

Aprovado para publicação em 16.06.2010.

Artigo publicado em 30.06.2010. 